\title{
Structural, Elastic, Thermodynamic and Anisotropic Properties of T14-Carbon under Pressure
}

\author{
Ming-Fei WeI ${ }^{a}$ And QI-Dong Zhang ${ }^{b, *}$ \\ ${ }^{a}$ College of Information and Control Engineering, \\ Xi'an University of Architecture and Technology, Xi'an 710055, PR China \\ ${ }^{b}$ School of Microelectronics, Xidian University, Xi'an 710071, PR China
}

Received: 02.04.2020 \& Accepted: 30.04.2020

Doi: 10.12693/APhysPolA.138.518 *e-mail: qdzhang@xidian.edu.cn

\begin{abstract}
As a three-dimensional (3D) metallic carbon, T14-carbon consisting of interlocking fused hexagons is predicted by first-principles calculations. In this work, an investigation of the structural, elastic, thermodynamic and anisotropic properties of T14-carbon under the pressures of up to $50 \mathrm{GPa}$ is conducted using density functional theory. When the applied pressure increases from $0 \mathrm{GPa}$ to $50 \mathrm{GPa}$, the elasticconstants, bulk modulus, Young's modulus and Poisson's ratio increase while the shear modulus decreases. T14-carbon has dynamic stability and ductility and its thermal conductivity changes slowly with pressure. The anisotropic studies of the bulk modulus, shear modulus, Young's modulus and other parameters show that T14-carbon exhibits mechanical anisotropy, with maximum and minimum elastic anisotropy in the (100) and (111) planes according to Young's modulus.
\end{abstract}

topics: T14-carbon, first-principles calculations, elastic properties, anisotropic properties

\section{Introduction}

A significant challenge at present is to find new materials with excellent physical properties, which leads to high requirements for computational material design. Theoretical methodologies for structure prediction have recently made significant progress, as demonstrated by the important discoveries of novel solid materials with interesting physical properties. From age-old graphite and diamond to more recent C60 fullerene [1], 1D nanotubes [2] and 2D graphene $[3,4]$, carbon displays an amazing array of physical properties and plays an important role in science and technology. As a unique element, carbon can adopt a series of different structures, ranging from superhard insulators to ultrasoft semimetals and even superconductors. Carbon has the ability to adopt $s p, s p^{2}$ and $s p^{3}$ hybridized states, thus forming a wide range of allotropes. Many superhard carbon phases have been proposed such as H-carbon [5], monoclinic M-carbon [6], P222 1 -carbon [7], $\mathrm{P} 2 / \mathrm{m}_{54}$ [8], Cm-carbon [9], S-carbon [10], W-carbon [11], monoclinic $\mathrm{C}_{32}$ [12], Z-carbon [13], and R-carbon [14], and all are stable. Using first-principles methods, two novel superhard 3D $s p^{3}$ hybridized carbon allotropes $\left(\mathrm{Cmmm}-\mathrm{C}_{32}\right.$ and $\left.\mathrm{P} 6 / \mathrm{mmm} \mathrm{C}_{54}\right)$ were proposed by Zhang et al. [15]. They have honeycomb structures that are potential semiconductor device materials with the hardnesses of 83.72 and $54.01 \mathrm{GPa}$. Sheng et al. [16] predicted T-carbon with a lower density $\left(1.50 \mathrm{~g} / \mathrm{cm}^{3}\right)$ that could serve as a semiconductor with a direct band gap of $\approx 3.0 \mathrm{eV}$ and the hardness of $\approx 61.1 \mathrm{GPa}$. The structural, mechanical, and dynamical properties of T-II carbon were extensively studied by Li et al. [17]. T-II carbon can be derived by stacking two T-carbons together. This creates a semiconductor with a band gap of $0.88 \mathrm{eV}$ and has a higher hardness $(27.0 \mathrm{GPa})$ than that of T-carbon (5.6 GPa), using Chen's hardness model [18].

Among the varied research topics in this field, metal carbon has attracted considerable attention. Unfortunately, the synthesis and characterization of metal carbon structures are very challenging. In 1D system, armchair carbon nanotubes (CNTs) are metallic but it is difficult to separate them from other semiconductor structures in the synthesis process [19]. In 2D graphene, the $\pi$-bonding of the $p_{Z}$ orbital makes the sheet metallic-like in a plane but the DOS (density of states) at the Fermi level is zero [20]. Therefore, graphene is semimetallic and needs defects to make it into a metal [21]. The synthesis of 3D metallic carbon under environmental conditions remains a difficult problem. Itoh et al. [22] discovered a metallic carbon allotrope called the $\mathrm{K}_{4}$ phase but an analysis of its phonon dispersion and mechanical properties showed that it is both dynamically and mechanically unstable. $\mathrm{Bu}$ et al. [23] proposed a stable metallic carbon allotrope (Hex-C24) composed of $s p^{2}$ and $s p^{3}$ hybridized carbon atoms. Its hardness 
was estimated to exceed $44.5 \mathrm{GPa}$, approximately half of that of diamond. Zhang et al. [24] predicted the existence of a unique metallic T6 carbon consisting of interlocking hexagons. Unlike the previously studied $\mathrm{K}_{4}$ and the simple cubic high pressure metallic phases, it is stable under environmental conditions. Zhou et al. [25] studied the pressure effects on the mechanical and electronic properties of $\mathrm{C}_{14}$ by first-principles calculations. The results showed that metallic $\mathrm{C}_{14}$ is mechanically and dynamically stable under high pressure.

To further improve the stability and metal properties, Zhang et al. [24] designed a structure by replacing the hexagon units in T6-carbon with fused hexagons, which contain 14 atoms in the primitive cell and are labelled T14-carbon (referred to as T14 below). The structural, electronic and mechanical properties of T14 have been predicted preliminarily under pressure but the elastic, thermodynamic and other properties have rarely been investigated in detail. In this work, the structural, elastic, thermodynamic and anisotropic properties of T14 at different pressures are studied systematically.

\section{Theory and computational details}

The structural optimization and property predictions of T14 were performed utilizing plane wave pseudopotential density functional theory (DFT) [26] with a local density approximation (LDA) [27] and the generalized gradient approximation (GGA) parameterized by Perdew, Burke and Ernzerhof (PBE) [28], which were implemented in the Cambridge Serial Total Energy Package (CASTEP) code [29]. The Broyden-FletcherGoldfarb-Shanno (BFGS) [30] minimization scheme was used in structural optimizations. The $k$-point grid in the Brillouin zone was obtained from the Monkhorst-Pack method [31]. In the structure calculations, convergence tests were considered using a plane wave basis with an energy cutoff of $400 \mathrm{eV}$. The electron-ion interactions were described by ultrasoft pseudopotentials [32]. The phonon spectra were calculated by using density functional perturbation theory (DFPT) [33]. The self-consistent convergence of the total energy was $5 \times 10^{-6} \mathrm{eV} /$ atom, the maximum force on the atom was $0.01 \mathrm{eV} / \AA$, the maximum ionic displacement was within $5 \times 10^{-4} \AA$, and the maximum stress was within $0.02 \mathrm{GPa}$. The bulk and shear moduli were derived from the Voigt-Reuss-Hill averaging scheme [34]. All theoretical calculations were carried out under hydrostatic pressure which ranged from 0 to $50 \mathrm{GPa}$ with a step of $10 \mathrm{GPa}$.

\section{Results and discussion}

In a conventional cell of T14 (space group $\mathrm{P} 4_{2} / \mathrm{mmc}$, tetragonal system), there are fourteen carbon atoms. The crystal structure information of T14 can be found in [24]. The lattice parameters $a$
TABLE I

The lattice constants $a, c$, unit cell volume $V$ and density $\rho$ of T14 under pressure.

\begin{tabular}{c|c|c|c|c|c}
\hline \hline & $\begin{array}{c}\text { Preassure } \\
{[\mathrm{GPa}]}\end{array}$ & $\begin{array}{c}a \\
{[\AA]}\end{array}$ & $\begin{array}{c}c \\
{[\AA]}\end{array}$ & $\begin{array}{c}V \\
{\left[\AA^{3}\right]}\end{array}$ & $\begin{array}{c}\rho \\
{\left[\mathrm{g} / \mathrm{cm}^{3}\right]}\end{array}$ \\
\hline T14 & 0 & 2.591 & 14.560 & 95.420 & 2.926 \\
& 10 & 2.558 & 14.417 & 94.363 & 2.959 \\
& 20 & 2.529 & 14.345 & 91.767 & 3.043 \\
& 30 & 2.502 & 14.284 & 89.448 & 3.122 \\
& 40 & 2.478 & 14.227 & 87.358 & 3.196 \\
& 50 & 2.456 & 14.176 & 85.476 & 3.267 \\
\hline Diamond & 0 & 3.566 & & & 3.518 \\
& Exp. [56] & 3.567 & & & 3.516
\end{tabular}

and $c$, the unit of cell volume $V$ and the density $\rho$ of T14 under pressure in the range of $0-50 \mathrm{GPa}$ were calculated and are illustrated in Table I. The calculated lattice constants of diamond with the GGA level are consistent with the experimental values (see Table I), so the results of T14 obtained from the GGA level are credible in this paper. Also further results are obtained with GGA.

To analyze the structural change, lattice constant ratios $a / a_{0}, b / b_{0}$, and $c / c_{0}$ and volume ratio $V / V_{0}$ of T14 were calculated at pressures from 0 to $50 \mathrm{GPa}$ (where $a_{0}, b_{0}, c_{0}$ and $V_{0}$ are lattice constants and volume at zero pressure and temperature equilibrium, respectively). In addition, this change was compared with other carbon allotropes, and the results are plotted in Fig. 1. We can see that the $a / a_{0}$ decreases more rapidly than the $c / c_{0}$ of T14 which suggests that the $a$-axis is more compressible than the $c$-axis. From the $a / a_{0}$ value, the incompressibility of $\mathrm{T} 14$ is smaller than that of $\mathrm{C}_{96}$ but slightly weaker than that of diamond while the $c / c_{0}$ of the incompressibility of T14 is greater than that of diamond. The volume ratio $V / V_{0}$ of $\mathrm{T} 14$ decreases with increasing applied pressure and changes between $\mathrm{C}_{96}$ and diamond. This result predicts that the incompressibility of $\mathrm{T} 14$ is greater than that of $\mathrm{C}_{96}$, slightly greater than that of c-BN but slightly weaker than that of diamond and P222 $2_{1}$-carbon [7].

Moreover, it is seen in Fig. 1a that the compression of the $c$-axis of T14 is less than that of the $a$-axis of diamond. We can analyse the reason behind it from the point of view of the $\mathrm{C}-\mathrm{C}$ bond length. There are four different $\mathrm{C}-\mathrm{C}$ bonds in $\mathrm{T} 14$, while diamond only has one $\mathrm{C}-\mathrm{C}$ bond. The crystal structure of T14 is shown in Fig. 1c. The red sphere is the $\mathrm{C} 1$ atom, the blue sphere is the $\mathrm{C} 2$ atom, the green sphere is the C3 atom and the orange sphere is the $\mathrm{C} 4$ atom. The four different carbon atom positions can be depicted, i.e., C1: $4 \mathrm{~h}$ $(0.5,0.5,0.95204), \mathrm{C} 2: 4 \mathrm{i}(0.5,0.0,0.90235), \mathrm{C} 3: 4 \mathrm{i}$ $(0.5,0.0,0.80796)$, and C4: $2 \mathrm{f}(0.5,0.5,0.25)$. The four different $\mathrm{C}-\mathrm{C}$ bonds in $\mathrm{T} 14$ are $d_{1}$ between $\mathrm{C} 1$ and $\mathrm{C} 2, d_{2}$ between $\mathrm{C} 2$ and $\mathrm{C} 3, d_{3}$ between $\mathrm{C} 3$ and $\mathrm{C} 4$, and $d_{4}$ between $\mathrm{C} 4$ and $\mathrm{C} 4$. For T14, the compression amount of the $a$-axis and $b$-axis 

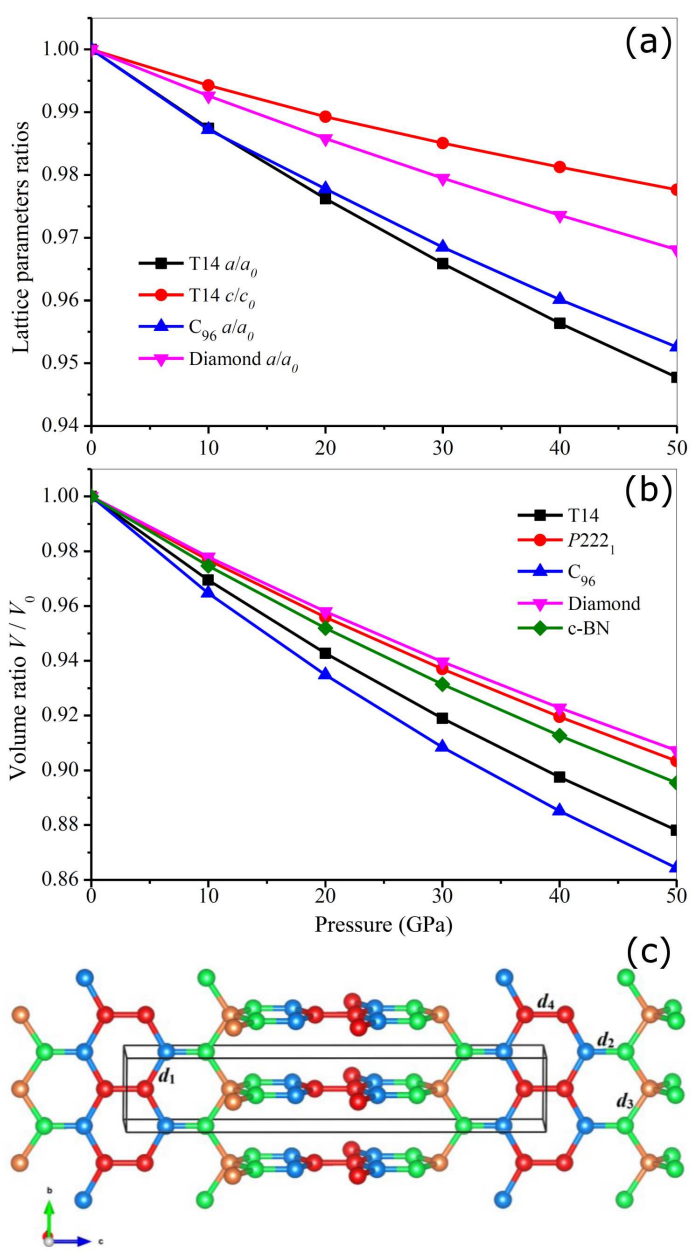

Fig. 1. (a) Lattice constants ratios of $\mathrm{T} 14, \mathrm{C}_{96}$ and diamond as a function of pressure, (b) the volume ratio $V / V_{0}$ of $\mathrm{T} 14, \mathrm{C}_{96}, P 222_{1}$-caron, c-BN and diamond as a function of pressure. The crystal structure of T14 (c).

is the same (because $a=b$ ), and along the $a$-axis and $b$-axis directions there are only $d_{1}$ and $d_{3}$ contributions. On the $c$-axis, all four $\mathrm{C}-\mathrm{C}$ bonds appear in this direction. When the pressure increased from 0 to $50 \mathrm{GPa}$, the bond lengths of $d_{2}$ and $d_{4}$ decreased from $1.36868 \AA$ and $1.39078 \AA$ to $1.34160 \AA$ and $1.36515 \AA$, respectively, which was a $1.98 \%$ and $1.84 \%$ decrease relative to zero pressure. In turn, the bond lengths of $d_{1}$ and $d_{3}$ decreased from $1.48235 \AA$ and $1.54422 \AA$ to $1.41278 \AA$ and $1.47689 \AA$, respectively, which was a $4.69 \%$ and $4.36 \%$ decrease relative to zero pressure.

For the $\mathrm{C}-\mathrm{C}$ bond length of diamond, the bond length decreased from $1.54410 \AA$ to $1.49495 \AA$ which was a $3.18 \%$ decrease relative to zero pressure. Since the decrease in bond length of $d_{2}$ and $d_{4}$ in T14 is smaller than that of diamond, the compression of the $c$-axis in T14 is also smaller than that of diamond.

The elastic properties of solids are very important [35-44] and they are not only closely related to various basic solid-state phenomena, such as

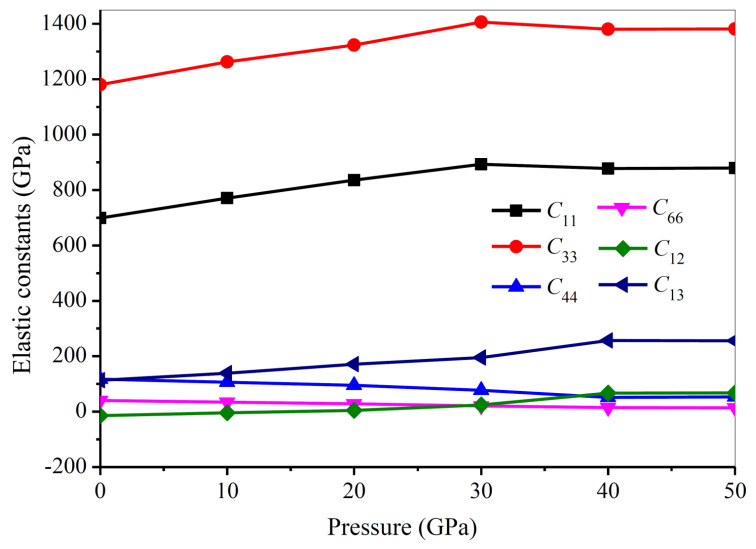

Fig. 2. Elastic constants of T14 as a function of pressure.

inter-atomic bonding, state equations and phonon spectra but also related to specific heat, thermal expansion, Debye temperature and other thermodynamic parameters. In addition, elastic constants are essential for many practical applications relevant to the mechanical properties of solids. For tetragonal symmetry crystals, there are six independent elastic constants $\left(C_{11}, C_{33}, C_{44}, C_{66}, C_{12}\right.$ and $\left.C_{13}\right)$. The mechanical stability criteria of tetragonal symmetry are given as follows [45]: $C_{11}>0, C_{33}>0$, $C_{44}>0, C_{66}>0, C_{11} C_{12}>0, C_{11}+C_{33}>2 C_{13}$, and $2\left(C_{11}+C_{12}\right)+C_{33}+4 C_{13}>0$.

The calculated results of the elastic constants of T14 at different pressures are listed in Table II and the change trend is shown in Fig. 2. Most of the elastic constants $C_{i j}$ increase with growing pressure. The elastic constants $C_{11}, C_{22}$ and $C_{33}$ represent the stiffness of the material when the stress is applied along the directions of [100], [010] and [001], respectively. The results suggest $C_{11}=C_{22}<C_{33}$, which means that the anti-deformation ability along the [100] and [010] directions is similar but lower than that along the [001] direction under pressure. The other elastic constants, such as $C_{44}, C_{66}, C_{12}$ and $C_{13}$, are all affected by pressure. The obtained elastic constants satisfy the stability criterion which indicates that the mechanical stability of T14 reaches $50 \mathrm{GPa}$. The elastic constants of $\mathrm{C}_{96}$, M-carbon and diamond at $0 \mathrm{GPa}$ are presented in Table II. Note that $C_{11}$ and $C_{33}$ of T14 are smaller than those of diamond and larger than those of $\mathrm{C}_{96}$ and consequently the incompressibility in the [100] and [001] directions of T14 is weaker than that of diamond and stronger than that of $\mathrm{C}_{96}$. The value of $C_{11}$ for $\mathrm{T} 14$ is smaller than that of M-carbon but $C_{33}$ has opposite value. Thus, the incompressibility in the [001] direction of T14 and M-carbon is basically similar. To confirm the stability of T14 under high pressure, the phonon spectra at $50 \mathrm{GPa}$ were calculated. As shown in Fig. 3, there is no imaginary frequency in the entire Brillouin zone. Therefore, T14 is dynamically stable at $50 \mathrm{GPa}$. 
TABLE II

Calculated elastic constants $C_{i j}$ (in GPa) of T14, compared with $\mathrm{C}_{96}$, M-carbon and diamond under pressure.

\begin{tabular}{c|c|c|c|c|c|c|c}
\hline \hline & $\begin{array}{c}\text { Pressure } \\
{[\mathrm{GPa}]}\end{array}$ & $C_{11}$ & $C_{33}$ & $C_{44}$ & $C_{66}$ & $C_{12}$ & $C_{13}$ \\
\hline T14 carbon & 0 & 698.64 & 1180.87 & 117.41 & 40.10 & -14.75 & 112.97 \\
& 10 & 770.55 & 1263.03 & 106.55 & 34.77 & -3.86 & 138.78 \\
& 20 & 835.75 & 1323.36 & 95.02 & 28.04 & 4.62 & 171.15 \\
& 30 & 892.99 & 1406.80 & 77.42 & 20.45 & 24.35 & 194.88 \\
& 40 & 878.12 & 1380.93 & 51.10 & 14.34 & 66.87 & 256.39 \\
& 50 & 879.83 & 1381.40 & 53.61 & 14.22 & 67.45 & 255.72 \\
\hline C96 & 0 & 636 & & 263 & & 89 & \\
\hline Miamond & 0 & 1017 & 1009 & 433 & 455 & 77 & 116 \\
\hline
\end{tabular}

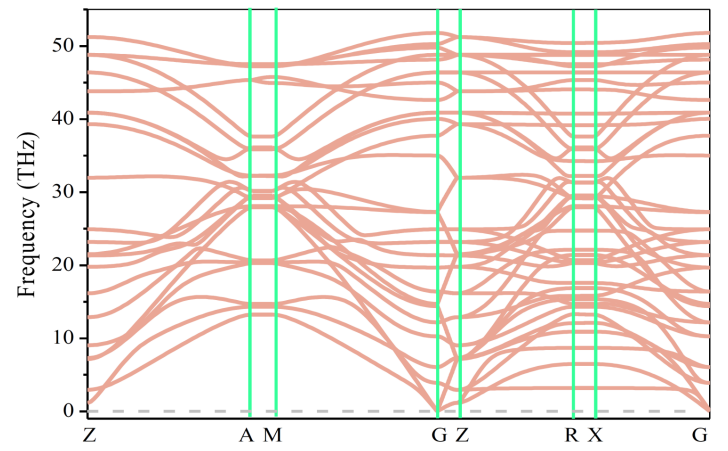

Fig. 3. Phonon spectra of T14 at $50 \mathrm{GPa}$.

The bulk modulus $B$ and shear modulus $G$ are used to measure the resistance of reversible deformation to a volume change under an applied pressure and shear stress, respectively. From the calculated elastic constants, the bulk modulus $B_{H}$ and the shear modulus $G_{H}$ can be estimated according to the Voigt-Reuss-Hill (VRH) averaging scheme. For tetragonal symmetry, the computational formulas are as follows [45]:

$$
\begin{aligned}
B_{\mathrm{V}} & =\frac{1}{9}\left[2\left(C_{11}+C_{12}\right)+C_{33}+4 C_{13}\right] \\
B_{\mathrm{R}} & =\frac{C^{2}}{M}, \\
G_{\mathrm{V}} & =\frac{1}{30}\left(M+3 C_{11}-3 C_{12}+12 C_{44}+6 C_{66}\right),
\end{aligned}
$$

$$
G_{\mathrm{R}}=15\left[\frac{18 B_{\mathrm{V}}}{C^{2}}+\frac{6}{C_{11}-C_{12}}+\frac{6}{C_{44}}+\frac{3}{C_{66}}\right]^{-1}
$$

$$
M=C_{11}+C_{12}+2 C_{33}-4 C_{13}
$$$$
C^{2}=\left(C_{11}+C_{12}\right) C_{33}-2 C_{13}^{2}
$$

$G_{\mathrm{H}}=\frac{G_{\mathrm{R}}+G_{\mathrm{V}}}{2}$,

$B_{\mathrm{H}}=\frac{B_{\mathrm{R}}+B_{\mathrm{V}}}{2}$.
Young's modulus is a physical quantity that describes the ability of solid materials to resist deformation and depends only on the physical properties of the material itself [46]. Young's modulus $E$ is used to measure the stiffness of a solid, defined as the ratio of stress to strain. Moreover, Poisson's ratio $\nu$ provides more information about the properties of bond forces than any other elastic constant. Young's modulus $E$ and Poisson's ratio $\nu$ are obtained from the following formulas [47]:

$$
\begin{aligned}
& E=\frac{9 B_{\mathrm{H}} G_{\mathrm{H}}}{3 B_{\mathrm{H}}+G_{\mathrm{H}}}, \\
& v=\frac{1}{2} \frac{3 B_{\mathrm{H}}-2 G_{\mathrm{H}}}{3 B_{\mathrm{H}}+G_{\mathrm{H}}} .
\end{aligned}
$$

The calculated values of the bulk modulus $B_{\mathrm{H}}$, shear modulus $G_{\mathrm{H}}$ and Young's modulus $E$ at different pressures are shown in Table III and the change tendency as a function of pressure is depicted in Fig. 4a. The bulk modulus and Young's modulus increase while the shear modulus decreases at pressures from 0 to $50 \mathrm{GPa}$. The values of the bulk modulus are much larger than those of the shear modulus which means that T14 tends to resist volume change better than shape change.

To predict the ductility and brittleness of the material, Pugh [48] proposed the ratio of the bulk to shear modulus $(B / G)$ as an indicator. The bulk modulus represents the flexural strength and the shear modulus represents the plastic deformation strength. The higher $B / G$ is, the better the ductility while the lower $B / G$ is, the greater the brittleness. According to the Pugh criterion, the critical value that distinguishes ductility from brittleness is $\approx 1.75$. The material shows ductility if $B / G>1.75$, otherwise - the material is brittle. In addition, Poisson's ratio $\nu$ is consistent with $B / G$, thus the larger Poisson's ratio $\nu(\nu>0.26)[49]$ is, the better the ductility is. When the applied pressure increases from $0 \mathrm{GPa}$ to $50 \mathrm{GPa}$, the $B_{\mathrm{H}} / G_{\mathrm{H}}$ and $\nu$ parameters of T14 are calculated and shown in Table III. The characteristic change trend is depicted in Fig. 4b. In the region $B_{\mathrm{H}} / G_{\mathrm{H}}>1.75$, the obtained trend suggests 
TABLE III

Calculated bulk modulus $B_{\mathrm{H}}$ (in GPa), shear modulus $G_{\mathrm{H}}$ (in GPa), Young's modulus $E$ (in GPa), the ratio $B_{\mathrm{H}} / G_{\mathrm{H}}$, Poisson's ratio $\nu$, anisotropy factors $A_{B}, A_{G}$ and $A^{U}$ of T14 under pressure.

\begin{tabular}{c|c|c|c|c|c|c}
\hline \hline $\begin{array}{c}\text { Pressure } \\
{[\mathrm{GPa}]}\end{array}$ & 0 & 10 & 20 & 30 & 40 & 50 \\
\hline$B_{\mathrm{V}}$ & 333.39 & 372.39 & 409.85 & 446.78 & 477.39 & 477.65 \\
$B_{\mathrm{R}}$ & 301.52 & 339.65 & 375.92 & 411.51 & 437.66 & 438.30 \\
$G_{\mathrm{V}}$ & 212.80 & 218.27 & 220.15 & 220.30 & 193.81 & 195.10 \\
$G_{\mathrm{R}}$ & 105.63 & 95.20 & 81.37 & 63.10 & 43.95 & 44.44 \\
$B_{\mathrm{H}}$ & 317.45 & 356.02 & 392.89 & 429.14 & 457.52 & 457.97 \\
$G_{\mathrm{H}}$ & 159.20 & 156.74 & 150.75 & 141.70 & 118.88 & 119.77 \\
$E$ & 409.20 & 410.05 & 400.97 & 382.96 & 328.21 & 330.50 \\
$B_{\mathrm{H}} / G_{\mathrm{H}}$ & 1.994 & 2.271 & 2.606 & 3.029 & 3.849 & 3.824 \\
$\nu$ & 0.285 & 0.308 & 0.330 & 0.351 & 0.380 & 0.380 \\
$A_{B}$ & 0.050 & 0.046 & 0.043 & 0.041 & 0.043 & 0.043 \\
$A_{G}$ & 0.337 & 0.393 & 0.460 & 0.555 & 0.630 & 0.629 \\
$A^{U}$ & 5.179 & 6.560 & 8.618 & 12.542 & 17.140 & 17.041
\end{tabular}
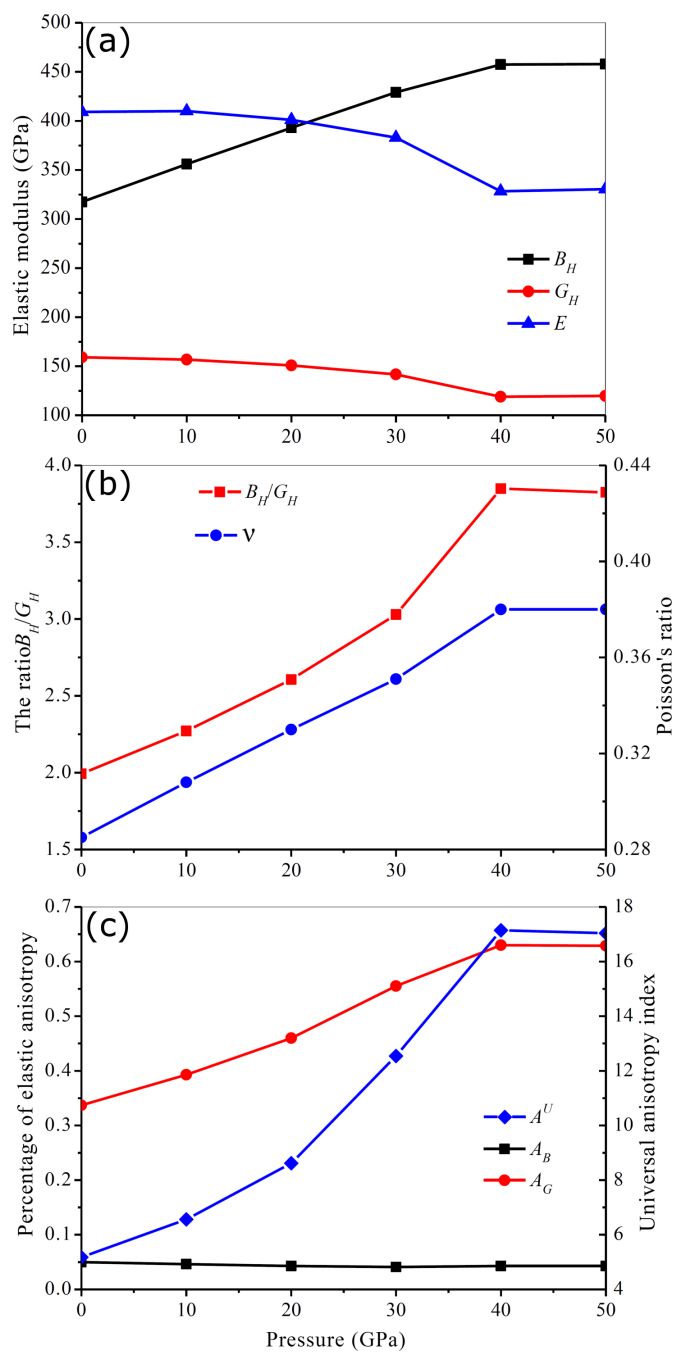

Fig. 4. (a) Elastic modulus $B_{\mathrm{H}}, G_{\mathrm{H}}$ and $E$ of T14 as a function of pressure, (b) the ratio $B_{\mathrm{H}} / G_{\mathrm{H}}$ and Poisson's ratio $\nu$ as a function of pressure, (c) the percentage of elastic anisotropy $a_{B}, A_{G}$ and universal anisotropy index $A^{U}$ as a function of pressure. that $\mathrm{T} 14$ is a ductile material and the ductility improves with increasing pressure. In the meantime, Poisson's ratio $\nu>0.26$ also implies that the material is ductile and the change is basically consistent with $B_{\mathrm{H}} / G_{\mathrm{H}}$.

To investigate the thermodynamic properties of T14, the Debye temperature $\Theta_{\mathrm{D}}$ and the sound velocities were calculated. The Debye temperature is one of the fundamental parameters for solid materials and is correlated with many physical properties, such as thermal expansion, melting point, specific heat and thermal conductivity [50]. The Debye temperature reflects the temperature of the normal vibration mode of the crystal. According to [51], a higher Debye temperature means stronger normal vibration and better thermal conductivity. The Debye temperature $\Theta_{D}$ and sound velocity can be calculated by $[52,53]$ :

$$
\Theta_{\mathrm{D}}=\frac{h}{k}\left(\frac{3 n}{4 \pi} \frac{N_{\mathrm{A}} \rho}{M}\right)^{\frac{1}{3}} v_{m},
$$

where $h$ represents Planck's constant, $k_{\mathrm{B}}$ represents the Boltzmann constant, $N_{\mathrm{A}}$ represents the Avogadro number, $n$ represents the number of atoms in the molecule, $M$ represents the molecular weight, and $\rho$ represents the density. The average sound velocity $v_{m}$ is calculated from

$$
v_{m}=\left[\frac{1}{3}\left(\frac{2}{v_{t}^{3}}+\frac{1}{v_{l}^{3}}\right)\right]^{\frac{1}{3}}
$$

where $v_{t}$ and $v_{l}$ are the transverse and longitudinal sound velocities, respectively, which can be obtained from Navier's equation [52]:

$$
\begin{aligned}
& v_{l}=\sqrt{\frac{1}{\rho}\left(B+\frac{4 G}{3}\right)}, \\
& v_{t}=\sqrt{\frac{G}{\rho}} .
\end{aligned}
$$

The calculated results of the transverse, longitudinal and average sound velocities and the Debye 
TABLE IV

Calculated sound velocity $v_{t}, v_{l}, v_{m}$, and Debye temperature $\Theta_{\mathrm{D}}$ of $\mathrm{T} 14$ under pressure.

\begin{tabular}{c|c|c|c|c}
\hline \hline $\begin{array}{c}\text { Pressure } \\
{[\mathrm{GPa}]}\end{array}$ & $v_{t}[\mathrm{~m} / \mathrm{s}]$ & $v_{l}[\mathrm{~m} / \mathrm{s}]$ & $v_{m}[\mathrm{~m} / \mathrm{s}]$ & $\Theta_{\mathrm{D}}[\mathrm{K}]$ \\
\hline 0 & 7376 & 13454 & 8223 & 1292 \\
10 & 7278 & 13818 & 8138 & 1283 \\
20 & 7039 & 13971 & 7892 & 1256 \\
30 & 6737 & 14071 & 7576 & 1216 \\
40 & 6099 & 13883 & 6886 & 1114 \\
50 & 6055 & 13751 & 6924 & 1129
\end{tabular}

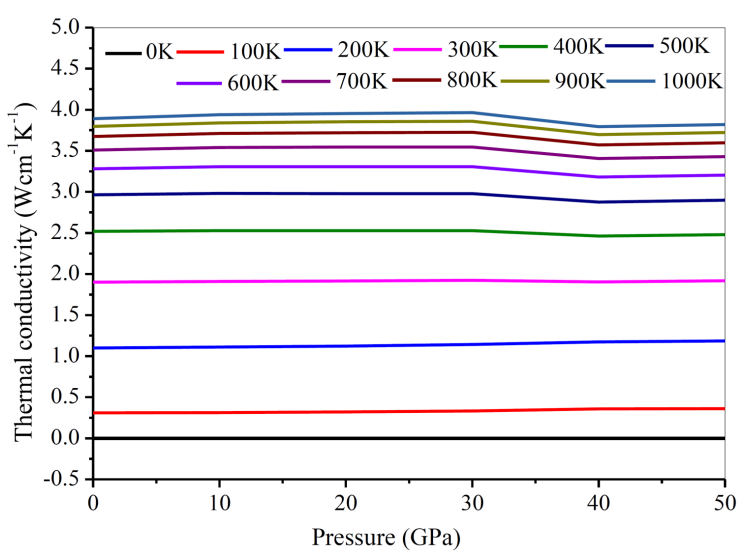

Fig. 5. Thermal conductivity of T14 as a function of pressure.

temperature for T14 are presented in Table IV. The Debye temperature is $1292 \mathrm{~K}$ for $\mathrm{T} 14$ at $T=0 \mathrm{~K}$ and $P=0 \mathrm{GPa}$ and changes by $12.62 \%$ in the pressure range of $0-50 \mathrm{GPa}$.

The thermal conductivity of materials is usually characterized by the coefficient of thermal conductivity $(\kappa)$ which is a measure of a substance's ability to conduct heat. The material is an excellent thermal conductor with a large $\kappa$ and a poor thermal conductor with a small $\kappa$. The changes in $\kappa$ with pressure at different temperatures are plotted in Fig. 5. When the pressure changes from $0 \mathrm{GPa}$ to $50 \mathrm{GPa}, \kappa$ does not change obviously. In other words, the thermal conductivity of T14 is slightly related to the pressure.

The anisotropy of the crystal means that the periodicity and density of atoms are different along different directions of the lattice which leads to different physical and chemical properties of the crystal in different directions. Therefore, anisotropy is of great significance in engineering science and crystal physics. There are many different ways to describe the elastic anisotropy which can be characterized from the bulk modulus, shear modulus, Young's modulus and other parameters. The structural change of the crystal is due to variations in the lattice constants $a, b$ and $c$ at various pressures. Therefore, the anisotropy is different because of the variations of the elastic constants with pressure. The anisotropy of the bulk modulus along the $a$-axis and $c$-axis with respect to the $b$-axis can be estimated by:

$$
\begin{aligned}
& A_{B a}=\frac{B_{a}}{B_{b}}, \\
& A_{B c}=\frac{B_{c}}{B_{b}} .
\end{aligned}
$$

Note that $a$ value of 1.0 indicates elastic isotropy and any departure from 1.0 represents elastic anisotropy, $B_{a}, B_{b}$ and $B_{c}$ are the bulk moduli along the $a, b$ and $c$ axes, which can be calculated by [50]:

$$
\begin{aligned}
& B_{a}=a \frac{\mathrm{d} P}{\mathrm{~d} a}=\frac{\Lambda}{1+\alpha+\beta}, \\
& B_{b}=b \frac{\mathrm{d} P}{\mathrm{~d} b}=\frac{B_{a}}{\alpha}, \\
& B_{c}=c \frac{\mathrm{d} P}{\mathrm{~d} c}=\frac{B_{a}}{\beta},
\end{aligned}
$$

with

$$
\begin{aligned}
\Lambda & =C_{11}+2 C_{12} \alpha+2 C_{13} \beta \\
& +C_{22} \alpha^{2}+C_{33} \beta^{2}+2 C_{23} \alpha \beta, \\
\alpha & =\left[\left(C_{11}-C_{12}\right)\left(C_{33}-C_{13}\right)\right. \\
& \left.-\left(C_{23}-C_{13}\right)\left(C_{11}-C_{13}\right)\right] \\
& \times\left[\left(C_{33}-C_{13}\right)\left(C_{22}-C_{12}\right)\right. \\
& \left.-\left(C_{13}-C_{23}\right)\left(C_{12}-C_{23}\right)\right]^{-1} \\
\beta & =\left[\left(C_{22}-C_{12}\right)\left(C_{11}-C_{13}\right)\right. \\
& \left.-\left(C_{11}-C_{12}\right)\left(C_{23}-C_{12}\right)\right] \\
& \times\left[\left(C_{22}-C_{12}\right)\left(C_{33}-C_{13}\right)\right. \\
& \left.-\left(C_{12}-C_{23}\right)\left(C_{13}-C_{23}\right)\right]^{-1}
\end{aligned}
$$

The calculated $B_{a}, B_{b}$ and $B_{c}$ at different pressures are shown in Table V. For diamond $B_{a}=B_{b}=$ $B_{c}=1124.33 \mathrm{GPa}$ which is smaller than that of T14, namely $B_{c}=1707.74$ GPa (see Fig. 1a). Obviously, all the values increase as the pressure increases within 0-50 GPa. In fact, the bulk modulus is the largest along the $c$-axis but the smallest along the $a$-axis ( $b$-axis). These results indicate that the compression resistance increases gradually and the compressibility is the smallest along the $c$-axis but the largest along the $a$-axis ( $b$-axis). The linear bulk modulus $A_{B a}$ remains constant and the fluctuation of $A_{B c}$ is approximately $11.74 \%$ at different pressures. These results clearly suggest that T14 is elastic and anisotropic.

The shear anisotropic factors provide a measure of the degree of anisotropy in the bonding between atoms in different planes. The shear anisotropic factor for the (100) shear plane between the [011] and [010] directions is denoted as $A_{1}$, the shear 
Linear bulk modulus $B_{a}, B_{b}, B_{c}$, anisotropy factors $A_{B a} A_{B c}, A_{1}, A_{2}$ and $\mathrm{A}_{3}$ of $\mathrm{T} 14$ under pressure.

\begin{tabular}{c|c|c|c|c|c|c|c|c}
\hline \hline $\begin{array}{c}\text { Pressure } \\
{[\mathrm{GPa}]}\end{array}$ & $B_{a}[\mathrm{GPa}]$ & $B_{b}[\mathrm{GPa}]$ & $B_{c}[\mathrm{GPa}]$ & $A_{B a}$ & $A_{B c}$ & $A_{1}$ & $A_{2}$ & $A_{3}$ \\
\hline 0 & 732.34 & 732.34 & 1707.74 & 1 & 2.332 & 0.284 & 0.284 & 0.112 \\
10 & 827.07 & 827.07 & 1900.96 & 1 & 2.298 & 0.243 & 0.243 & 0.090 \\
20 & 914.35 & 914.35 & 2115.22 & 1 & 2.313 & 0.209 & 0.209 & 0.067 \\
30 & 1002.18 & 1002.18 & 2302.13 & 1 & 2.297 & 0.162 & 0.162 & 0.047 \\
40 & 1043.53 & 1043.53 & 2715.10 & 1 & 2.602 & 0.117 & 0.117 & 0.035 \\
50 & 1046.29 & 1046.29 & 2702.34 & 1 & 2.583 & 0.123 & 0.123 & 0.035 \\
& \\
&
\end{tabular}

For isotropic crystals, the $A_{B}, A_{G}$ and $A^{U}$ are all zero and the deviation from zero indicates the degree of elastic anisotropy. Unlike all other anisotropy measures, the deviation of $A^{U}$ from zero defines the extent of crystal anisotropy and accounts for both the shear modulus and the bulk modulus contributions. Thus, $A^{U}$ represents a universal measure to quantify the crystal elastic anisotropy. The calculated anisotropy factors under pressures are shown in Table III and plotted in Fig. 4c. The $A_{B}$ value is small and fluctuates with pressure which implies that T14 has slight anisotropy in the bulk modulus. The $A_{G}$ value is larger than $A_{B}$, indicating that the shear modulus is more dependent on direction than the bulk modulus. In addition, the $A^{U}$ increases monotonically with increasing pressure which means that $\mathrm{T} 14$ has more obvious anisotropy with increasing pressure.

The maximum and minimum results of Young's modulus in different planes of T14 are presented in Table VI. Young's modulus of the (001) plane, (010) plane, (011) plane, (100) plane, (101) plane, (110) plane and (111) plane are calculated in the pressure range of $0-50 \mathrm{GPa}$. At $0 \mathrm{GPa}$, the maximum Young's modulus is $1143.55 \mathrm{GPa}$, the minimum is $143.06 \mathrm{GPa}$, and the maximum $E_{\max } / E_{\min }$ ratio is 7.99 which is much higher than that of diamond silicon (1.57). As seen from the maximum values, the variations of the (001), (011) and (101) planes are approximately the same, and the variations of the (011), (100) and (110) planes are also the same. They all have a tendency to increase first and then decrease slightly but the variation of the (111) plane decreases oppositely. The variations in the (001), (110) and (111) planes and the (010) and (100) planes and the (011) and (101) planes are the same as the minimum values, which all have a basic tendency to decrease. The $E_{\max } / E_{\min }$ ratio on all planes generally moves upward and the ordering of (100) plane $>(001)$ plane $>(010)$ plane $>$ $(010)$ plane $=(100)$ plane $>(111)$ plane implies the maximum and minimum elastic anisotropy of T14 in the (100) and (111) planes. As a valid method 
TABLE VI

The maximum values, minimum values, and the ratio $E_{\max } / E_{\min }$ of Young's modulus in different planes for T14 under pressure.

\begin{tabular}{c|c|c|c|c|c|c|c|c|c|c|c}
\hline \hline $\begin{array}{c}\text { Pressure } \\
{[\mathrm{GPa}]}\end{array}$ & \multicolumn{3}{|c|}{$E_{\max }[\mathrm{GPa}]$} & \multicolumn{3}{c|}{$E_{\min }[\mathrm{GPa}]$} & \multicolumn{5}{c}{$E_{\max } / E_{\min }$} \\
\hline & $(001)$ & $(010)$ & $(111)$ & $(001)$ & $(010)$ & $(011)$ & $(010)$ & $(011)$ & $(001)$ & $(110)$ & $(111)$ \\
& $(011)$ & $(100)$ & & $(110)$ & $(100)$ & $(101)$ & $(100)$ & $(101)$ & & & \\
& $(101)$ & $(110)$ & & $(111)$ & & & & & & & \\
\hline 0 & 686.89 & 1143.55 & 379.57 & 143.06 & 376.78 & 191.88 & 3.04 & 3.58 & 4.80 & 7.99 & 2.65 \\
10 & 754.82 & 1212.79 & 356.42 & 127.07 & 354.87 & 172.11 & 3.42 & 4.39 & 5.94 & 9.54 & 2.80 \\
20 & 813.24 & 1253.65 & 327.35 & 104.80 & 326.54 & 145.88 & 3.84 & 5.57 & 7.76 & 11.96 & 3.12 \\
30 & 865.98 & 1324.01 & 275.83 & 78.11 & 275.47 & 111.85 & 4.81 & 7.74 & 11.09 & 16.95 & 3.53 \\
40 & 830.07 & 1241.80 & 189.23 & 55.49 & 189.12 & 77.93 & 6.57 & 10.65 & 14.96 & 22.38 & 3.41 \\
50 & 832.01 & 1243.34 & 197.79 & 55.05 & 197.67 & 78.61 & 6.29 & 10.58 & 15.11 & 22.58 & 3.59
\end{tabular}
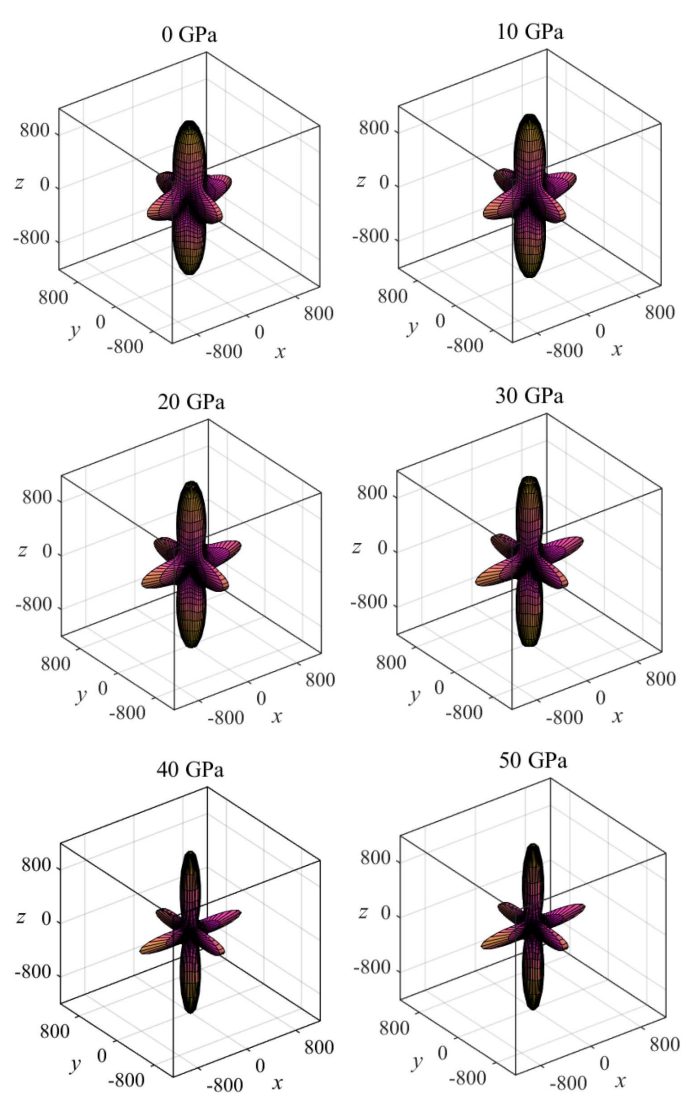

Fig. 7. The directional dependence of Young's modulus for T14 on given pressure value.

to describe the elastic anisotropic behaviour of a crystal completely, the 3D surface constructions of the directional dependencies of Young's modulus are plotted in Fig. 7. In accordance with the previous analysis, the elastic anisotropy is more obvious with increasing pressure.

\section{Conclusions}

The structural, elastic, thermodynamic and anisotropic properties of T14 under pressures of up to $50 \mathrm{GPa}$ were investigated systematically. As the pressure increases, the elastic constants $C_{11}, C_{33}$, $C_{12}$ and $C_{13}$ increase, and the $a$-axis is more compressible than the $c$-axis. The bulk modulus and Young's modulus increase while the shear modulus decreases. Phonon spectra prove that T14 is dynamically stable at $50 \mathrm{GPa}, B / G>1.75$, Poisson's ratio $\nu>0.26$ and the material is ductile. The Debye temperature is $1292 \mathrm{~K}$ at $0 \mathrm{GPa}$ and the thermal conductivity does not change obviously under pressure. When the applied pressure changes from $0 \mathrm{GPa}$ to $50 \mathrm{GPa}$, the anisotropy is calculated and analysed from different aspects such as the bulk modulus, shear modulus and Young's modulus. The anisotropy of the bulk modulus changes by $11.74 \%$ along the $c$-axis with respect to the $b$-axis and the shear anisotropic factors of the (100), (010) and (001) shear planes decrease by $56.7 \%, 56.7 \%$ and $68.8 \%$, respectively. The percentage of elastic anisotropy for the shear modulus $A_{G}$ is larger than that of the bulk modulus $A_{B}$ which is small and fluctuates, and the general anisotropy index $A^{U}$ increases monotonically. In general, the maximum values of Young's modulus in most planes increase but minimum values decrease. The ratio of $E_{\max } / E_{\min }$ in all researched planes basically increases with increasing pressure and the maximum and minimum elastic anisotropy of T14 are in the (100) and (111) planes on the basis of Young's modulus.

\section{Acknowledgments}

This research was funded by the Key Research and Development Plan of Shaanxi Province, China, grant number 2017ZDXM-GY-001.

\section{References}

[1] H.W. Kroto, J.R. Heath, S.C. O'Brien, R.F. Curl, R.E. Smalley, Nature 318, 162 (1985).

[2] S. Iijima, T. Ichihashi, Nature 363, 603 (1993). 
[3] K.S. Novoselov, A.K. Geim, S.V. Morozov, D. Jiang, Y. Zhang, S.V. Dubonos, I.V. Grigorieva, A.A. Firsov, Science 306, 666 (2004).

[4] W. Zhang, C.C. Chai, Q.Y. Fan, Y.X. Song, Y.T. Yang, ChemNanoMat 6, 139 (2020).

[5] C.Y. He, L.Z. Sun, C.X. Zhang, X.Y. Peng, K.W. Zhang, J.X. Zhong, Solid State Commun. 152, 1560 (2012).

[6] Q. Li, Y.M. Ma, A.R. Oganov, H.B. Wang, H. Wang, Y. Xu, T. Cui, H.K. Mao, G.T. Zou, Phys. Rev. Lett. 102, 175506 (2009).

[7] X.X. Zhang, Y.C. Wang, J. Lv, C.Y. Zhu, Q. Li, M. Zhang, Q. Li, Y.M. Ma, J. Chem. Phys. 138, 114101 (2013).

[8] X.Z. Li, M.J. Xing, Mater. Chem. Phys. 242, 122480 (2020).

[9] X.Z. Li, M.J. Xing, Comp. Mater. Sci. 143, 32 (2018).

[10] D. Li, K. Bao, F.B. Tian, Z.W. Zeng, Z. He, B.B. Liu, T. Cui, Phys. Chem. Chem. Phys. 14, 4347 (2012).

[11] J.T. Wang, C. Chen, Y. Kawazoe, Phys. Rev. Lett. 106, 075501 (2011).

[12] X.C. Yang, Q. Wei, B. Wei, H.Y. Yan, R.K. Yang, M.G. Zhang, Q.H. Chen, X.M. Wang, R.H. Yao, C.Y. Zhao, C.Y. Ding, Acta Phys. Pol. A 136, 940 (2019).

[13] Z.S. Zhao, B. Xu, X.F. Zhou, L.M. Wang, B. Wen, J.L. He, Z.Y. Liu, Y.J. Tian, Phys. Rev. Lett. 107, 215502 (2011).

[14] H. Niu, X.Q. Chen, S. Wang, D. Li, W.L. Mao, Y. Li, Phys. Rev. Lett. 108, 135501 (2012).

[15] W. Zhang, C.C. Chai, Q.Y. Fan, Y.X Song, Y.T. Yang, J. Appl. Phys. 126, 145704 (2019).

[16] X.L. Sheng, Q.B. Yan, F. Ye, Q.R. Zheng, G. Su, Phys. Rev. Lett. 106, 155703 (2011).

[17] D. Li, F.B. Tian, D.F. Duan, Z.L. Zhao, Y.X. Liu, B.H. Chu, X.J. Sha, L. Wang, B.B. Liu, T. Cui, $R S C A d v$. 4, 17364 (2014).

[18] X.Q. Chen, H.Y. Niu, D.Z. Li, Y.Y. Li, Intermetallics 19, 1275 (2011).

[19] M. Ouyang, J.L. Huang, C.L. Cheung, C.M. Lieber, Science 292, 702 (2001).

[20] K.S. Novoselov, A.K. Geim, D. Jiang, I.V. Grigorieva, S.V. Morozov, S.V. Dubonos, A.A. Firsov, M.I. Katsnelson, Nature 438, 197 (2005).
[21] J. Lahiri, Y. Lin, P. Bozkurt, I.I. Oleynik, M. Batzill, Nat. Nanotechnol. 5, 326 (2010).

[22] M. Itoh, M. Kotani, H. Naito, T. Sunada, Y. Kawazoe, T. Adschiri,Phys. Rev. Lett. 102, 055703 (2009).

[23] H.X. Bu, M.W. Zhao, W.Z. Dong, S.W. Lu, X.P. Wang, J. Mater. Chem. C 2, 2751 (2014).

[24] S.H. Zhang, Q. Wang, X.S. Chen, P. Jena, PNAS 110, 18809 (2013).

[25] Y.J. Zhou, Q. Wei, B. Wei, R.K. Yang, K. Cheng, M.G. Zhang, H.Y. Yan, Q.H. Chen, J.Q. Zhang, Int. J. Mod. Phys. B 33, 1950193 (2019).

[26] W. Kohn, L.J. Sham, Phys. Rev. 140 A1133 (1965).

[27] J.P. Perdew, A. Zunger, Phys. Rev. B 23 , 5048 (1981).

[28] J.P. Perdew, K. Burke, M. Ernzerhof, Phys. Rev. Lett. 77, 3865 (1996).

[29] S.J. Clark, M.D. Segall, C.J. Pickard, P.J. Hasnip, M.I.J. Probert, K. Refson, M.C. Payne, Z. Kristallogr. 220, 567 (2005).

[30] B.G. Pfrommer, M. Côté, S.G. Louie, M.L. Cohen, J. Comput. Phys. 131, 233 (1997).

[31] H.J. Monkhorst, J.D. Pack, Phys. Rev. B 13, 5188 (1976).

[32] D. Vanderbilt, Phys. Rev. B 41, 7892 (1990).

[33] S. Baroni, S.D. Gironcoli, A.D. Corso, P. Giannozzi, Rev. Mod. Phys. 73, 515 (2001).

[34] R. Hill, Proc. Phys. Soc. Lond. Sect. A 65, 349 (1952).

[35] Q. Fan, Y. Zhao, X. Yu, Y. Song, W. Zhang, S. Yun, Diam. Relat. Mater. 106, 107831 (2020).

[36] Q.Y. Fan, H.Q. Wang, Y.X. Song, W. Zhang, S.N. Yun, Comput. Mater. Sci. 178, 109634 (2020).

[37] X.Z. Li, M.J. Xing, Comp. Mater. Sci. 158, 170 (2019).

[38] Q.Y. Fan, W.Z. Zhang, S.N. Yun, J. Xu, Y.X. Song, Chem. Eur. J. 24, 17280 (2018).

[39] Q.Y. Fan, R. Niu, W.Z. Zhang, W. Zhang, Y.C. Ding, S.N. Yun, ChemPhysChem 20, 128 (2019).

[40] Q Wei, Q Zhang, M.G. Zhang, H.Y. Yan, L.X. Guo, B. Wei, Front. Phys. 13, 136105 (2018).

[41] Q.Y. Fan, J. Xu, W.Z. Zhang, Y.X. Song, S.N. Yun.J. Appl. Phys. 126, 045709 (2019). 
[42] Q. Zhang, Y. Zou, Q. Fan, Y. Yang, Materials 13, 1280 (2019).

[43] W. Zhang, C. Chai, Q. Fan, Y. Song, Y. Yang, Materials 13, 1926 (2020).

[44] Q.Y. Fan, H.Q. Wang, W.Z. Zhang, M.F. Wei, Y.X. Song, W. Zhang, S.N. Yun, Curr. Appl. Phys. 19, 1325 (2019).

[45] Z.J. Wu, E.J. Zhao, H.P. Xiang, X.F. Hao, X.J. Liu, J. Meng, Phys. Rev. B 76, 054115 (2007).

[46] Q.Y. Fan, W.Z. Zhang, Y.X. Song, W. Zhang, S.N. Yun, Semicond. Sci. Technol. 35, 055012 (2020).

[47] Q.Y. Fan, C.C. Chai, Q. Wei, Y.T. Yang, L.P. Qiao, Y.B. Zhao, P.K. Zhou, M.J. Xing, J.Q. Zhang, R.H. Yao, Mater. Sci. Semicond. Proc. 40, 676 (2015).

[48] S.F. Pugh, Philos. Mag. 45, 823 (1954).

[49] J.J. Lewandowski, W.H. Wang, A.L. Greer, Philos. Mag. Lett. 85 , 77 (2005).
[50] P. Ravindran, F. Lars, P.A. Korzhavyi, B. Johansson, J. Wills, O. Eriksson, J. Appl. Phys. 84, 4891 (1998).

[51] Y.H. Duan, B. Huang, Y. Sun, M.J. Peng, S.G. Zhou, J. Alloys Comp. 590, 50 (2014).

[52] O.L. Anderson, J. Phys. Chem. Solids 24, 909 (1963).

[53] Q.Y. Fan, C.C. Chai, Q. Wei, H.Y. Yan, Y.B. Zhao, Y.T. Yang, X.H. Yu, Y. Liu, M.J. Xing, J.Q. Zhang, R.H. Yao, J. Appl. Phys. 118, 185704 (2015).

[54] D. Connétable, O. Thomas, Phys. Rev. B 79, 094101 (2009).

[55] Q.Y. Fan, Q. Wei, H.Y. Yan, M.G. Zhang, Z.X. Zhang, J.Q. Zhang, D.Y Zhang, Comp. Mater. Sci. 85, 80 (2014).

[56] M.L. Petrescu, Diam. Relat. Mater. 13, 1848 (2004). 